\title{
ANÁLISE DE DADOS ULTRASSÔNICOS PARA O RECONHECIMENTO DE VAZIOS NO INTERIOR DE PAINÉIS DE ALVENARIA MACIÇA
}

\author{
RODRIGUES, TÁLISSON \\ Aluno de Graduação \\ LAREB, Engenharia Civil,UFC \\ Ceará; Brasil \\ talisson.ecivil@alu.ufc.br
}

\author{
COSTA, CARLOS \\ Professor, Doutor \\ LAREB, UFC \\ Ceará; Brasil \\ carloshumberto@ufc.br
}

\author{
ARAUJO, EMANUEL \\ Aluno de Graduação \\ LAREB, Engenharia Civil,UFC \\ Ceará; Brasil \\ emanuhenriqcivil@gmail.com
}

PAZ, ROSINEIDE

Professora, Doutora

LAREB, UFC

Ceará; Brasil

rfpaz2@gmail.com

\author{
SOUSA, ISRAEL \\ Aluno de Graduação \\ LAREB, Engenharia Civil,UFC \\ Ceará; Brasil \\ israelnlsousa@gmail.com
}

\author{
MESQUITA, ESEQUIEL \\ Professor, Doutor \\ LAREB, UFC \\ Ceará; Brasil \\ emesquita@ufc.br
}

\section{RESUMO}

Nos últimos 40 anos, observa-se uma crescente preocupação com a estabilidade estrutural para minimizar os riscos à segurança, em especial nas construções históricas, pela alta complexidade construtiva e valor cultural. Logo, os ensaios não destrutivos, com foco nos ultrassônicos, surgem como ferramenta para caracterizar o estado das alvenarias. $\mathrm{O}$ presente estudo objetiva analisar experimentalmente a onda ultrassônica no interior de painéis de alvenaria maciça e observar variações do tempo de propagação na presença de vazios. Para tal, construiu-se dois painéis, um com condições construtivas ideais e o outro foi inserido aberturas interiores. Após o ensaio, utilizou-se métodos estatísticos para comparar o tempo de propagação da onda nos painéis. Os resultados mostram dispersão e variação na linha de tendência da média para o painel com vazios, espera-se que tais resultados contribuam para identificar os vazios nas alvenarias e sirvam para interpretações fiáveis de ensaios de campo.

Palavras-chave: Construções históricas, Ensaios não destrutivos, Métodos estatísticos, Interpretações

\begin{abstract}
Over the past 40 years, there has been a growing concern about structural stability to minimize security risks, especially in historic buildings due to their high constructive complexity and cultural value. Therefore, non-destructive tests, focusing on ultrasonic, emerge as a tool to characterize the state of masonry. The present study aims to experimentally analyze the ultrasonic wave inside solid masonry panels and to observe variations of propagation time in the presence of voids. For this, two panels were built, one with ideal constructive conditions and the other was inserted interior openings. After the test, statistical methods were used to compare the wave propagation time in the panels.. The results show dispersion and variation in the trend line from the mean to the void panel, it is expected that these results will help to identify the voids in the masonry and serve for reliable interpretations of field tests.
\end{abstract}

Keywords: Historical buildings, Non-destructive tests, Statistical methods, Interpretations. 


\section{INTRODUÇÃO}

Nos últimos 40 anos é crescente a preocupação a cerca da reabilitação e estabilidade estrutural das construções, a exemplo disso a elevação da realibitação frente as construções de novos edifícios, em destaque na Europa pode-se citar Portugal com o crescimento de $20 \%$ em construções não residenciais (FERREIRA; BRITO, 2007). As mudanças no entorno da edificação e as variáveis atmosféricas incidentes sobre a estrutura podem causar uma baixa na qualidade e vida útil dos materiais (DE SOUZA, et al. 2017). Corroborando com o autor, Lersch (2003) verifica a atuação conjunta de fatores ambientais e antropológicos na degradação do patrimônio histórico de Porto Alegre. A perca da capacidade resistente dos materiais ao serem submetidos as condições ambientais podem causar possíveis riscos à segurança; e se faz necessário medidas de conservação bem como reabilitação das estruturas.

As construções históricas demandam uma atenção especial por serem estruturas com valor cultural iminente, por apresentarem uma grande variedade de materiais constituintes, onde a complexidade e essa heterogeneidade acrescentam uma maior dificuldade para análise da condição e estado de segurança (ARÊDE; COSTA, 2002). Segundo Mesquita, et al. (2017) essas construções apresentam um grande valor cultural para o espaço e sociedade onde se encontram, fazendo-se necessária sua conservação.

Dentre as ferramentas para avaliação da condição estrutural, por sua grande possibilidade de reaplicação e conservação do meio analisado, os ensaios não destrutivos surgem como uma significativa ferramenta para analisar a estabilidade das construções. Conseguindo resultados satisfatórios tanto quando executados in-situ, como visto em Roque e Lourenço (2003) na caracterização do Centro Histórico de Bragança com aplicação do ensaio de macacos planos, e quando aplicados em situação controlada em laboratório como em Tavares (2006), no qual se aplica a termografia na análise de amostras em ambiente controlado que ajudaram no desenvolvimento de uma metodologia de campo.

Segundo Binda et al. (2000), os ensaios não-destrutivos podem ser muito úteis para a determinação das características de alvenarias históricas, conseguindo identificar vazios e fazer correlações para estimar parâmetros mecânicos, destacando-se como bastante promissores os ensaios ultrassônicos, radar e termografia. Dentre estes, o ensaio de ultrassom vem sendo empregado de forma abundante para estudos de alvenarias como visto em Mesquita et al. (2018), Alves (2017) e Wendrinch et al. (2006). Contudo, é notório uma lacuna no meio científico em estudos com foco no emprego de ferramentas estatísticas aplicadas e que contribuam de forma mais relevante com a análise dos componentes estruturais.

Diante do exposto, de modo a entender e interpretar de maneira fiável os ensaios ultrassônicos, o presente trabalho tem como objetivo analisar experimentalmente o comportamento da onda ultrassônica no interior de alvenarias maciças, observando-se as variações gerais do tempo de propagação na presença de vazios no meio com o auxílio de métodos estatísticos.

\section{ENSAIO EXPERIMENTAL}

Para a execução do experimento, foi utilizado o equipamento de ultrassom Pundit Lab-PROCEQ, com transdutores de $54 \mathrm{kHz}$. O processamento dos dados foi realizado no R Core Team (2019), com as ferramentas estatísticas descritas na Seção 3. Com o intuito de entender o comportamento da onda ultrassônica, buscou-se comparar uma situação ideal com um cenário induzido, onde esse segundo possui a presença de anomalias, que neste trabalho são os vazios. Para isso foram construídos dois painéis P1 e P2, com 1,50 m de altura, 1,00 m de largura e 13,5 cm de espessura, ambos com o mesmo material, nomeadamente tijolos maciços, de dimensões $4,6 \mathrm{~cm}$ x 24,7 cm x 12,3 cm, com massa específica de $2000 \mathrm{~kg} / \mathrm{m}^{3}$, tendo um dos lados revestidos completamente com argamassa de cal e areia (1:6), diferindo apenas na construção, pois no painel P2 foram deixados vazios em sua estrutura, como mostra a Figura 1. 

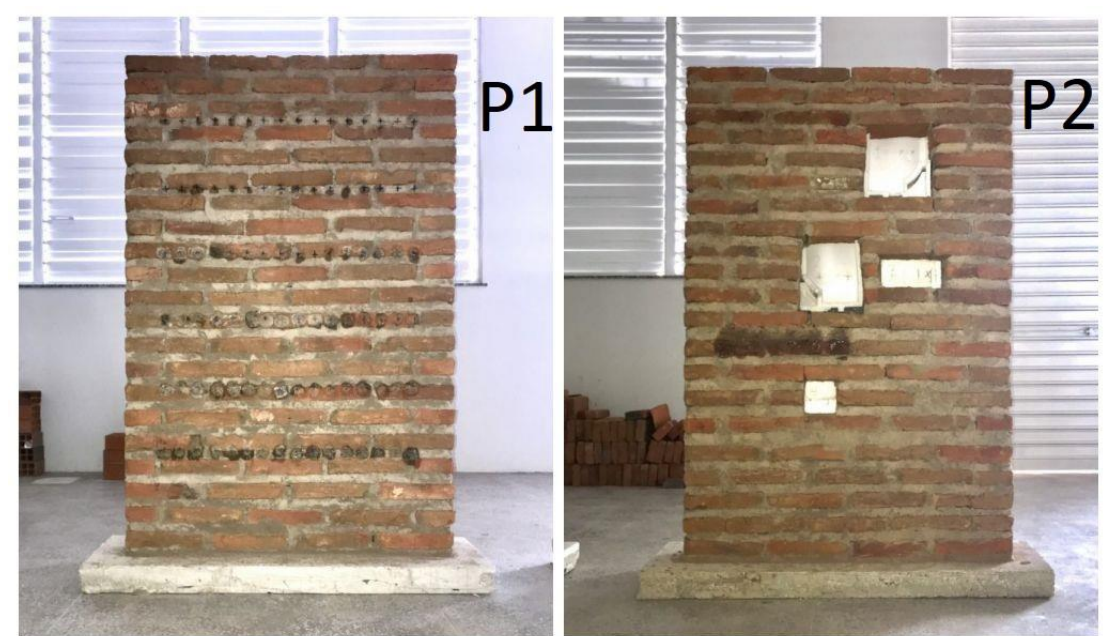

Figura 1: Painéis P1 e P2 de alvenaria maciça.

O método de medição escolhido foi o indireto, onde o transdutor emissor e o transdutor receptor ficam no mesmo plano no material (Figura 2). Essa configuração pode ser recomendada tanto em paredes com grandes espessuras, na qual o acesso a ambos os lados é impossibilitado, como também nas que apresentam pequenas espessuras onde a aplicação do método direto verificaria mais as capacidades individuais dos blocos, podendo não representar por si só as características do conjunto (ALVES, 2017).
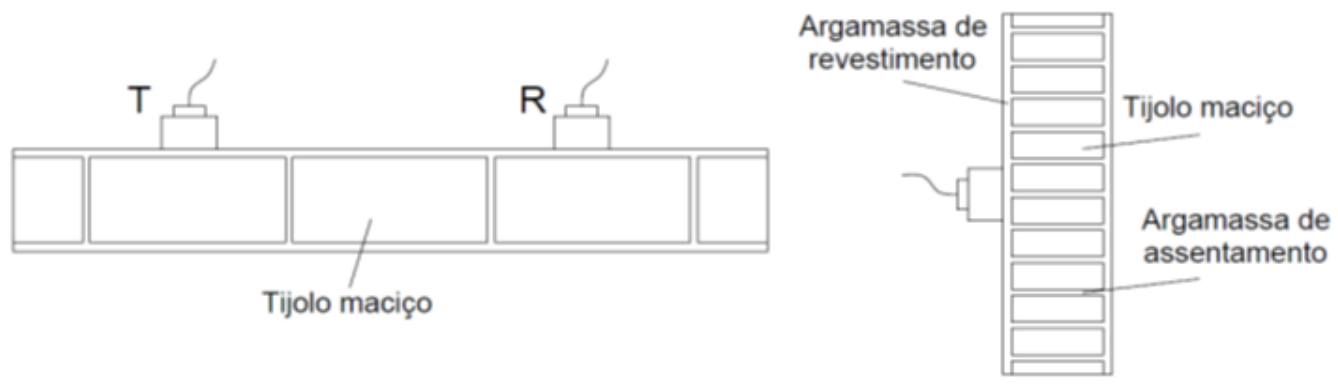

Figura 2: Esquema de medição ultrassônica pelo método indireto.

A aplicação do ensaio se deu na face com revestimento dos painéis. Para cada painel foram realizados testes ultrassônicos considerando as direções horizontal (X) e vertical (Y), com uma malha de quadrantes com dimensões de $20 \mathrm{~cm} \mathrm{X} 40 \mathrm{~cm}$, altura e largura respectivamente, no qual para a horizontal foi atribuído 0 para a posição onde ocorre a emissão da onda em cada quadrante. Em ambos os painéis foram distribuídos os quadrantes de modo a obter o máximo de quadros possíveis dentro das dimensões dos painéis, para isso deixou-se $10 \mathrm{~cm}$ a partir de cada borda do painel e 20 $\mathrm{cm}$ a partir da base de assentamento, objetivando separar essas regiões que são influenciadas de maneira diferente do resto do painel. A configuração final dispunha de dois quadrantes em cada uma das seis faixas de altura, totalizando doze quadros por painel, como é visto na Figura 3. 


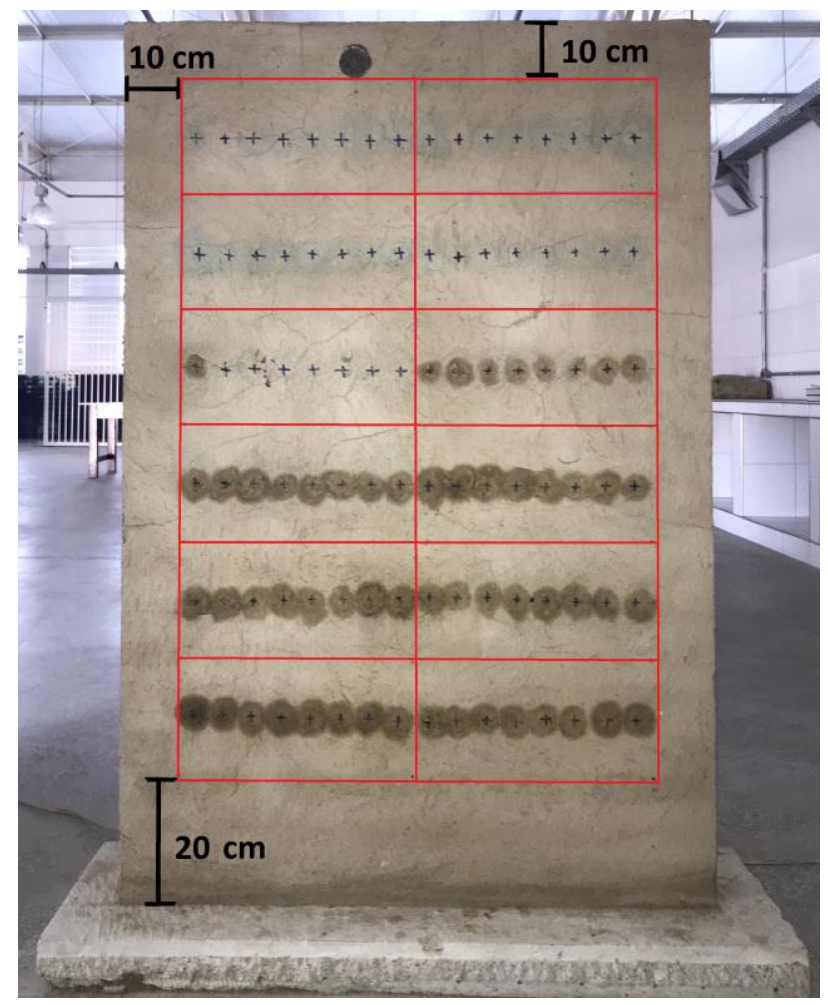

Figura 3: Painel dividido em quadrantes.

Cada quadrante (em cada painel) recebeu seis medições (distribuídas no centro), com os transdutores receptores espaçados entre si em cada ponto de X1 até X6. O transdutor emissor $E$ permanece estático enquanto se varia a posição do receptor em distâncias linearmente crescentes de propagação, onde para cada ponto foi medido o tempo de propagação da onda. A Figura 4 demostra o procedimento de medição nos quadrantes.

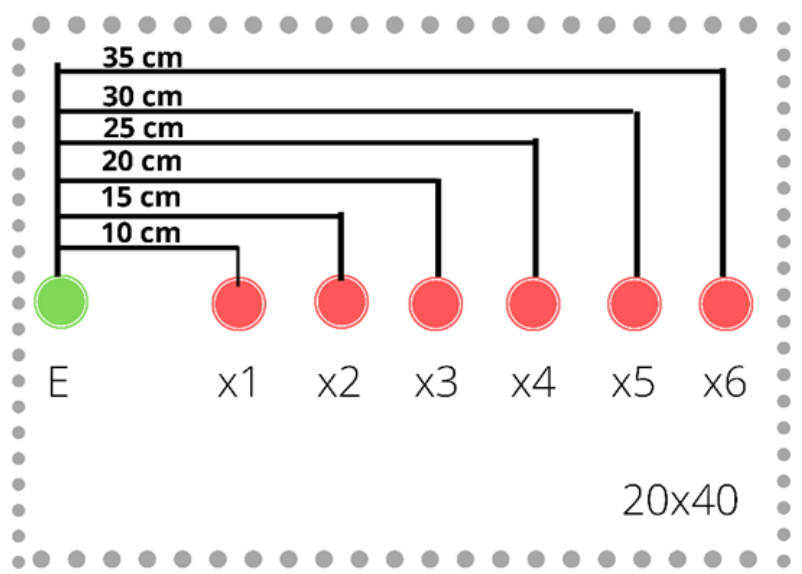

Figura 4: Esquema de demarcação dos pontos de mensuração do tempo de propagação da onda a partir de um ponto pré-fixado.

A metodologia de ensaio deste trabalho foi uma adaptação do que é preconizado na NBR-8802 (2019) e do que foi executado em Mesquita et al. (2018), de modo a adequar com as dimensões replicadas em laboratório, onde com quadrantes de $40 \mathrm{~cm}$, foram definidos seis pontos de medição espaçados em $5 \mathrm{~cm}$ e um ponto de emissão a $10 \mathrm{~cm}$ da primeira recepção, totalizando a largura do quadrante. Para cada posição mediu-se o tempo de propagação da onda, da sua emissão até sua recepção no transdutor. Vale ressaltar que para o emprego das ferramentas estatísticas foi escolhido trabalhar com o tempo e não com a velocidade para evitar transformações nos dados obtidos no ensaio. 


\section{FERRAMENTAS ESTATÍSTICAS PARA DADOS ULTRASSÔNICOS}

Nesta seção, ferramentas estatísticas são apresentadas como possíveis metodologias para a análise de dados ultrassônicos.

\subsection{Modelo misto para dados ultrassônicos}

Para uma exploração inicial dos dados ultrassônicos, o gráfico de perfil de cada quadrante foi plotado para os dois painéis conjuntamente. Esse tipo de gráfico é muito usado para analisar o comportamento das curvas fornecidas por medições repetidas em uma mesma unidade amostral. Em experimentos com medidas repetidas, existem várias unidades amostrais e as medidas são tomadas repetidamente em cada uma delas. Geralmente estas medições são realizadas ao longo do tempo e, nesse caso, são chamadas de estudo longitudinal.

Neste trabalho, as mensurações referem-se ao tempo em que a onda leva para ir do ponto de propagação até um outro ponto pré-fixado. Para cada unidade amostral, esse processo foi repetido seis vezes para seis pontos fixos igualmente espaçados entre si em uma linha reta. Para todas as seis medições, em uma mesma unidade amostral, a propagação foi emitida a partir de um mesmo ponto fixado seguindo a NBR-8802 (2019). O gráfico de perfil para os 24 quadrantes analisados, para os dois painéis, é mostrado em Figura 5. A partir desses perfis, como é esperado uma tendência linear para uma alvenaria em condições regulares ou com uma certa homogeneidade em sua composição, pode ser observada algum desvio da linearidade na tendência geral dos perfis? Caso seja observado algum desvio da linearidade, a tendência geral dos perfis de propagação indica anomalia na alvenaria?

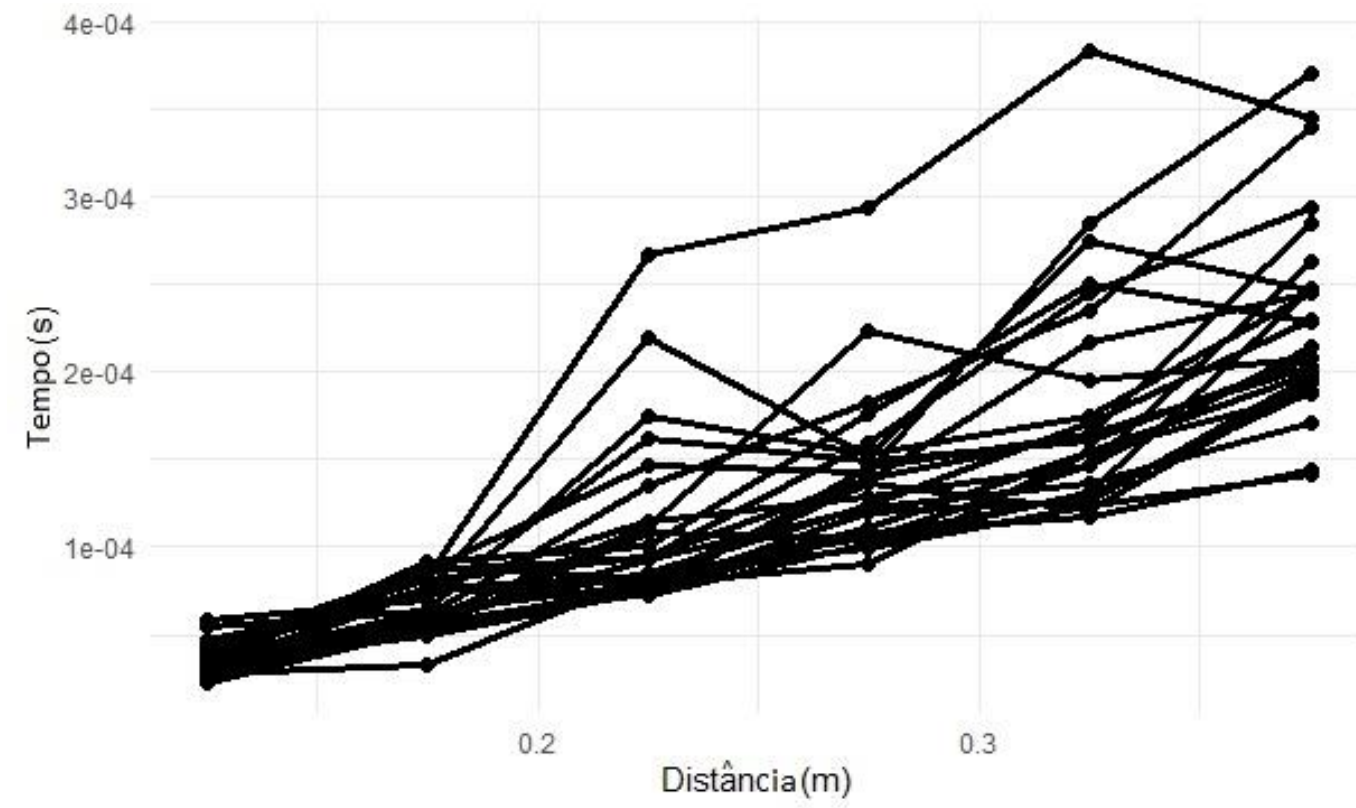

Figura 5: Gráfico do tempo obtido em cada ponto de medição de todos os quadrantes de ambos os painéis

Pelo exposto, uma abordagem que pode ser usada para analisar dados de propagação de pulso ultrassônicos é aquela normalmente usada para analisar dados longitudinais, ou seja, uma análise de regressão com efeito misto, em que o efeito de cada quadrante é levado em consideração na análise dos dados com a inclusão de um efeito aleatório. Para uma revisão do modelo misto, e para a introdução da metodologia para dados ultrassônicos, considere que cada tempo pode ser escrito em função da distância percorrida pela onda como:

$$
T_{i j}=f\left(D_{i j}, \boldsymbol{\beta}, \mathbf{b}_{i}\right)+\epsilon_{i j}
$$

em que o termo $\epsilon_{\mathrm{ij}}$ é nomeado erro aleatório considerado aqui como uma distribuição normal com média zero e variância constante $\sigma 2$, ou seja:

$$
\epsilon_{i j} \sim N\left(0, \sigma^{2}\right)
$$


A função $f\left(D_{i j}, \beta, \mathbf{b}_{i}\right)$ em (2) descreve a relação entre a variável tempo de propagação e a distância percorrida pela onda (como dito anteriormente, essa relação pode não ser linear, pode ser, por exemplo, uma curva polinomial de grau G, G> 1), nesta função, $\beta$ é o coeficiente que representa o efeito fixo (efeito global comum a todos os quadrantes) e $b_{i}$ é o coeficiente que representa o efeito aleatório, que leva em consideração o efeito do i-ésimo quadrante na tendência geral dos perfis.

O modelo de regressão com efeito aleatório talvez seja uma boa opção para modelar os dados ultrassônicos, porém é necessário um bom entendimento sobre qual é o significado da curva resultante da tendência global dos perfis. Se é esperado uma reta e tem-se um polinômio de grau 3, o que isso significa em termos de condições da alvenaria? Uma vez estabelecida a relação entre a tipo de curva resultado e uma dada anomalia em uma edificação, pode-se empregar modelos de classificação (ou clusterização) para tentar separar os quadrantes candidatos a investigações mais profundas, por fornecerem evidências de problemas em sua constituição.

Para que se tenha uma estimativa do efeito de cada quadrante na tendência global, deve-se adotar uma metodologia Bayesiana de estimação, em que é assumida uma distribuição de probabilidades a priori para todos os parâmetros que se deseja estimar. Mais detalhes sobre a estimativa Bayesiana para dados com medidas repetidas podem ser vistos em Broemeling (2015).

Para completar o modelo em (2) inserindo a distribuição adotada para o efeito aleatório, considere uma outra forma de escrever este mesmo modelo:

$$
\begin{aligned}
T_{i j} & \sim N\left(\mu_{i j}, \sigma^{2}\right) \\
\mu_{i j} & =f\left(D_{i j}, \boldsymbol{\beta}, \mathbf{b}_{i}\right) \\
\mathbf{b}_{i} & \sim N(0, \Sigma b),
\end{aligned}
$$

Em que $\Sigma$ b é a matriz de covariância dos efeitos aleatórios $\mathbf{b}_{\mathbf{i}}, \sigma^{2}$ a variância do termo de erro e $\mathrm{X} \sim \mathrm{N}(\mu, \Sigma \epsilon)$ denota uma variável aleatória que segue distribuição normal de probabilidades com média denotada por $\mu$ e variância (ou matriz de variância e covariância no caso multivariado) $\Sigma \epsilon$.

Para os dados ultrassônicos, um modelo misto foi ajustado considerando um polinômio de grau $\mathrm{G}=3$ para os efeitos fixos. Foi considerado um efeito aleatório apenas para o intercepto da curva (onde a curva cruza o eixo das ordenadas). Assim, tem-se:

$$
\begin{gathered}
T_{i j}=\mathrm{b}_{0}+\beta_{0}+\beta_{1} D_{i j}+\beta_{2} D^{2}{ }_{i j}+\beta_{2} D^{3}{ }_{i j}+\epsilon_{i j} \\
\mathrm{~b}_{0} \sim N\left(0, \sigma_{b}^{2}\right)
\end{gathered}
$$

Em que $\sigma^{2}{ }_{b}$ é a variância do intercepto aleatório. Note que o efeito aleatório poderia ser considerado, também, na forma da curva, ou seja, poderiam ser adotados efeitos aleatórios para a variável distância e suas variantes.

A fim de evitar correlação entre as variáveis explicativas $\left(D, D^{2}\right.$ e $\left.D^{3}\right)$, foi considerado o polinômio ortogonal dessas variáveis. A Tabela 1 mostra o efeito aleatório estimado do modelo e o intervalo de credibilidade de 95\%, que representam a influência de cada quadrante no modelo. Nesta tabela, podem ser observados cinco quadrantes da parede com anomalias com intervalos de credibilidade que não contêm zeros, ou seja, ao nível de $95 \%$ de confiança, pode-se dizer que esses quadrantes exercem influência no ajuste da curva. Em relação a parede sem anomalias, pode ser observado apenas um quadrante com efeito significativo.

Uma desvantagem em adotar um polinômio para modelar a tendência global de propagação de ondas é impor um comportamento que não necessariamente ocorre. Então, para uma exploração inicial desse comportamento, é importante considerar um método mais flexível. A próxima seção apresenta um método poderoso usado para modelar tendências sem impor uma função polinomial predefinida para todos os dados. Para esse método, uma regressão linear é considerada no modelo para cada conjunto de variáveis próximas umas das outras, levando a uma função flexível para todo o conjunto de dados. 
Tabela 1: Efeitos estimados dos quadrantes na curva polinomial $\mathrm{G}=3$ graus estimada e $95 \%$ de credibilidade.

\begin{tabular}{c|c|c|c|c|c|c}
\hline \multirow{2}{*}{ Quadrante } & \multicolumn{3}{|c|}{ Sem anomalia } & \multicolumn{3}{c}{ Com anomalia } \\
\cline { 2 - 7 } & Efeito médio & Lim $_{\text {inf }}$ & Lim $_{\text {sup }}$ & Efeito médio & Lim $_{\text {inf }}$ & Lim $_{\text {sup }}$ \\
\hline Q1 & 0,12 & $-0,05$ & 0,29 & 0,13 & $-0,03$ & 0,30 \\
\hline Q2 & 0,26 & 0,09 & 0,44 & 0,47 & 0,31 & 0,65 \\
\hline Q3 & $-0,07$ & $-0,24$ & 0,09 & $-0,07$ & $-0,23$ & 0,10 \\
\hline Q4 & 0,01 & $-0,16$ & 0,17 & 0,15 & $-0,01$ & 0,32 \\
\hline Q5 & 0,17 & 0,00 & 0,33 & 0,16 & 0,00 & 0,33 \\
\hline Q6 & $-0,17$ & $-0,34$ & 0,00 & $-0,25$ & $-0,43$ & $-0,08$ \\
\hline Q7 & $-0,16$ & $-0,33$ & 0,01 & $-0,15$ & $-0,32$ & 0,02 \\
\hline Q8 & $-0,07$ & $-0,24$ & 0,09 & 0,26 & 0,09 & 0,43 \\
\hline Q9 & 0,00 & $-0,17$ & 0,17 & $-0,21$ & $-0,38$ & $-0,04$ \\
\hline Q10 & 0,05 & $-0,11$ & 0,22 & $-0,34$ & $-0,50$ & $-0,17$ \\
\hline Q11 & $-0,09$ & $-0,26$ & 0,08 & $-0,16$ & $-0,33$ & 0,00 \\
\hline Q12 & $-0,05$ & $-0,22$ & 0,12 & 0,05 & $-0,12$ & 0,21 \\
\hline
\end{tabular}

\subsection{Método Loess para dados ultrassônicos}

Para explorar o tempo de propagação da onda, foi feito uma regressão ponderada suavizada local (chamada de método loess). O método loess foi proposto inicialmente por Cleveland, et al. (2017) e fornece ferramentas para ajuste de uma curva suavizada aos dados, usando para isto uma metodologia robusta. Este método é amplamente utilizado em geoestatística para construção de superfícies e é muito útil para explorar dados espaciais usando gráficos de superfícies ou de contorno. Aqui, esta técnica é usada para explorar a tendência das curvas de propagação de ondas por uso de uma suavização do gráfico de dispersão apoderado localmente (LOWESS).

Na construção de um gráfico pelo método LOWESS, um modelo de regressão linear é ajustado localmente usando o método dos mínimos quadrados. Ou seja, nesta abordagem, em cada ponto Dij uma regressão linear é ajustada usando mínimos quadrados ponderados, em que é dado mais peso às observações que estão localizadas mais próximas entre si. Assim, modelos de regressão lineares são considerados para um conjunto de observações da forma:

$$
T_{i}=\beta 0(D)+\beta 1(D) D_{i}+\epsilon_{i} \text {, para } D_{i} \in[D-h, D+h],
$$

Em que $T_{i}$ representa o i-ésimo tempo de propagação em uma dada distância $D_{i}$ e $\epsilon_{i}$ é o termo de erro para a i-ésima variável resposta (tempo), que é assumido como tendo uma distribuição normal com média zero e variância constante, para $\mathrm{i}=1,2, \cdots 144$. Em (4) h representa o tamanho da vizinhança em torno de D onde estão os pontos considerados para a regressão local. Então, para qualquer ponto D nos dados, a média da variável resposta é modelada linearmente em torno de D. Deste modo, aplica-se técnicas de regressão linear a uma fração dos dados. A vizinhança de D pode ser escolhida subjetivamente pelo especialista ou objetivamente pelos dados observados. Assim, muitas regressões lineares com apenas dois parâmetros ( $\beta 0$ (D) e $\beta 1$ (D)) podem ser ajustadas aos dados considerando "janelas" no plano cartesiano em torno de D.

Para reduzir o peso das contribuições de um ponto de dados $D_{i}$ que esteja mais longe de $\mathrm{D}$ que alguns outros, um esquema de peso pode ser adicionado ao modelo de regressão local, dado por (4). Isto consiste, basicamente, em estimar $\beta 0, \beta 1$ e $\sigma^{2}$ de modo a minimizar a versão ponderada do modelo dada por (4), ou seja, minimizar a soma:

$$
\Sigma\left\{\omega D\left(D_{i}\right)\left[T_{i}-\beta 0(D)-\beta 1(D) D_{i}\right]^{2}\right\}, \text { para } D_{i} \in[D-h, D+h] .
$$

Para isso, aqui é usado a função Kernel:

$$
K D(u)=\left(1-|u|^{3}\right)^{3}, \text { para }-1 \leq u \leq 1,
$$


Para definir os pesos $\omega D$ que são dados por:

$$
\omega_{D}(D i)=K_{D}\left(\underline{D_{i}}-\underline{-D}\right)
$$

Para uma exploração dos dados, as rotinas R foram usadas utilizando o pacote "ggplot2" disponibilizado por Wickham (2016), para traçar as curvas suavizadas a partir dos dados ultrassônicos. A Figura 6 mostra a curva suavizada para os perfis dos quadrantes separadamente para aqueles com anomalia e para aqueles sem anomalia.

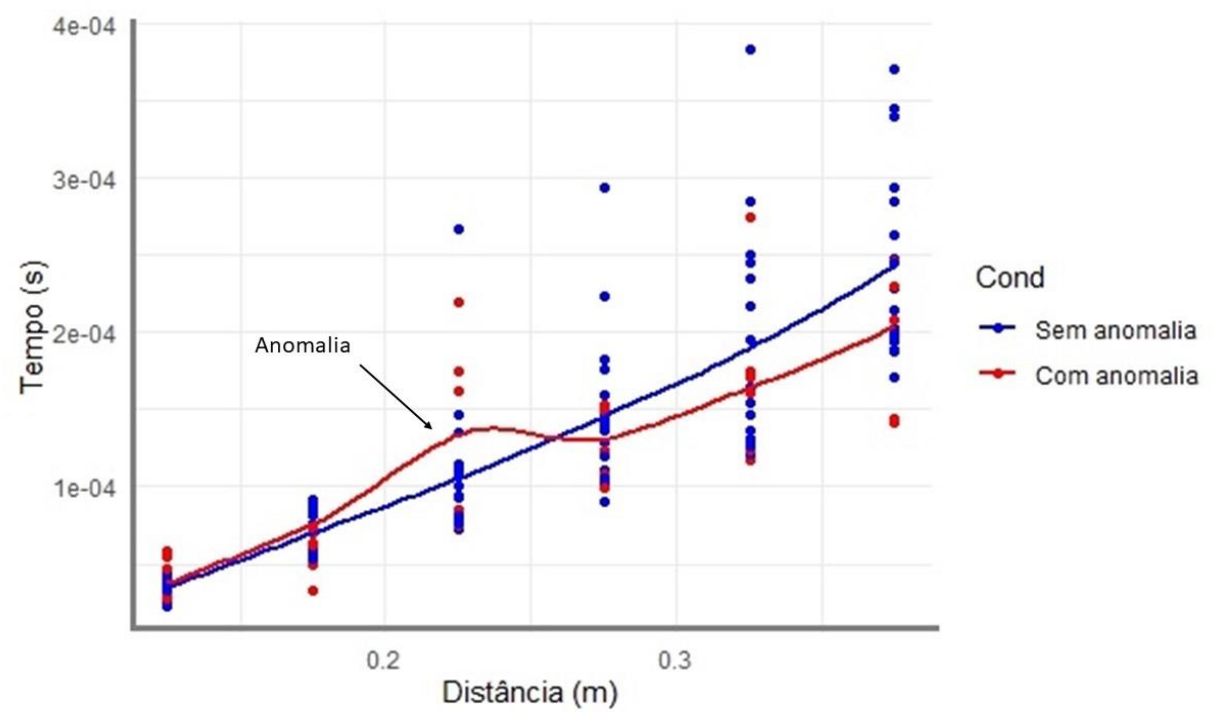

Figura 6: Gráfico do perfil dos dados ultrassônicos, considerando a transformação logarítmica para o tempo.

\section{RESULTADOS E DISCUSSÃO}

Neste trabalho foram utilizadas duas ferramentas estatísticas para fazer uma exploração dos dados ultrassônicos que foram observados a partir do experimento planejado, descrito na Seção 2. Um importante ponto a ser salientado é que para cada quadrante foram obtidas 6 medidas de tempo de propagação de ondas ultrassônicas que foram emitidas a partir do mesmo ponto. Como resultado, tem-se uma série de dados altamente correlacionados, não somente por serem observações obtidas da mesma alvenaria, mas também por serem observados a partir de ondas obtidas do mesmo ponto. Sendo assim, é esperado que exista uma correlação maior entre os pontos observados em cada quadrante do que entre aqueles que foram observados em quadrantes distintos, pois para cada dois pontos observados em quadrantes diferentes, temos pontos de propagação diferentes, além de serem pontos mais distantes entre si do que aqueles que estão no mesmo quadrante.

Assim, a fim de entender o comportamento do tempo de propagação da onda na alvenaria, foi exibido em um gráfico de perfis as curvas resultantes das medições de cada quadrante, considerando os dois painéis. Estas curvas podem ser vistas na Figura 1, em que pode ser observada a tendência linear e crescente esperada, uma vez que para percorrer uma distância maior a onda deve gastar, também, um tempo maior, pois foi medida no mesmo material. Analisando esta figura, não é possível perceber se existem perfis que não seguem a tendência linear crescente esperada, tão pouco é possível saber qual é a tendência linear que deveria ocorrer. No entanto, a partir de uma análise mais aprofundada, é possível descobrir se existem perfis que têm tendências que destoam do comportamento global. Para saber se existem tais perfis, foi ajustado um modelo de regressão polinomial misto, em que é modelado o efeito que cada quadrante exerce no intercepto da curva ajustada aos dados.

A Tabela 1 mostra estimativas desses efeitos, juntamente com intervalos de credibilidades que possibilitam tirar conclusões sobre se cada quadrante está ou não exercendo efeitos diferentes dos demais sobre o intercepto da curva. Caso o intervalo do efeito aleatório contenha o zero, tem-se evidências de que esse valor está muito próximo de zero, fazendo com que a distância da curva obtida a partir desse quadrante em relação a curva média global não seja significativa. Assim, os quadrantes que destoam em P2 (painel com anomalia) são Q2, Q6, Q8, Q9 e Q10, a Figura 7 mostra imagens de alguns quadrantes em que pode ser visto que os quadrantes Q2 e Q9 parecem não estarem afetados 
pelas anomalias da parede. Além disso, o painel sem anomalia, P2, também apresenta um quadrante com efeito significativo, a saber o Q2. Estes resultados, embora preliminares, sugerem que, mesmo impondo uma curva não muito flexível, é possível verificar quadrantes com anomalias influenciando na tendência geral.
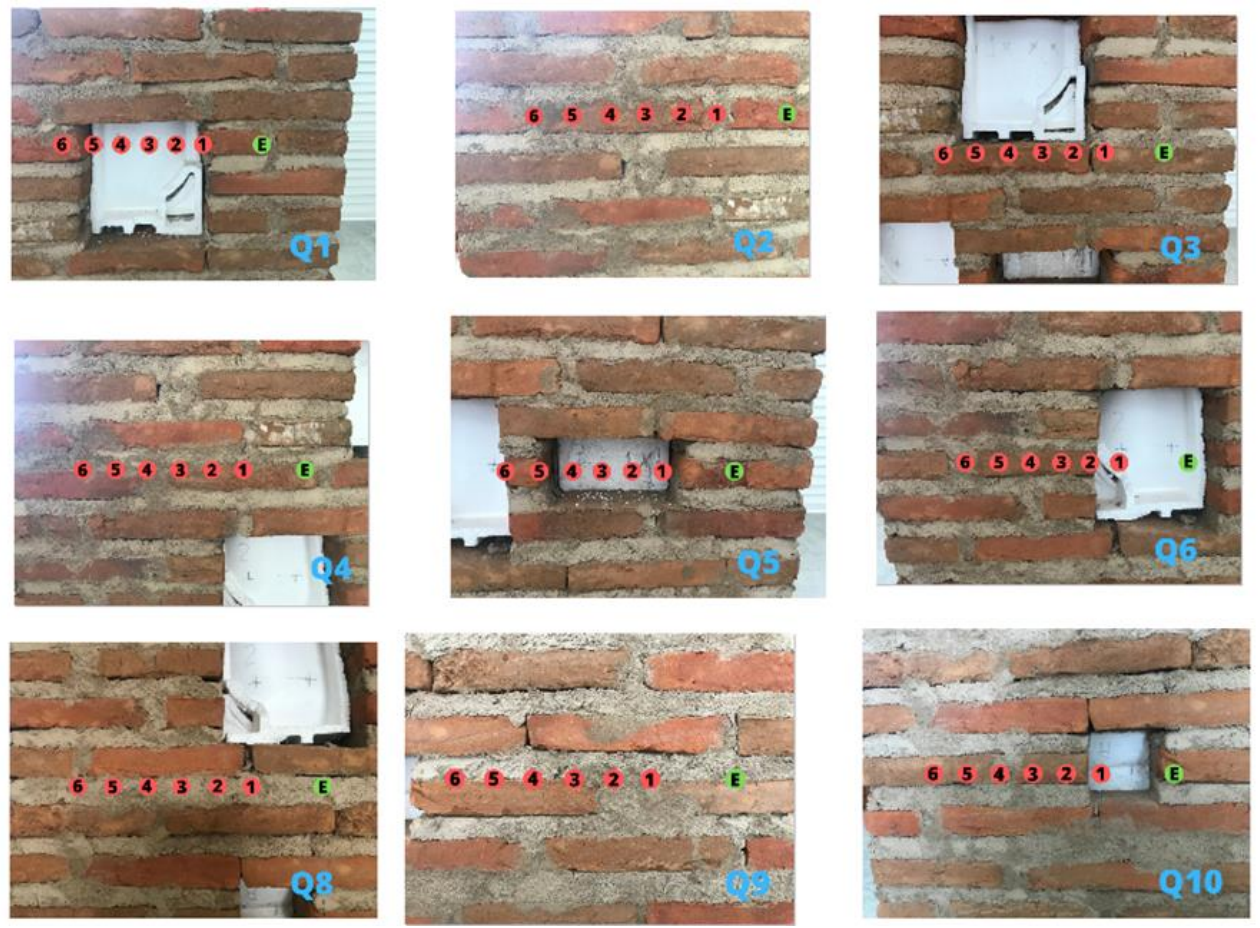

Figura 7: Alguns quadrantes do painel com anomalias.

Para se ter uma ideia da tendência global dos perfis, utilizou-se o método LOWESS para ajustar uma curva média aos dados separados por painéis. A Figura 8 mostra as duas curvas suavizadas, em que podem ser notados diferentes comportamentos para as duas tendências globais. Para o painel sem anomalias (sem vazios) observa-se uma tendência mais próxima da linear, enquanto para o painel com anomalias percebe-se uma mudança no comportamento da curva na altura da terceira medição, caracterizando-se uma perturbação. Essa diferença pode ser melhor percebida na Figura 6, mencionada anteriormente, em que se tem as duas curvas suavizadas plotadas uma sobre a outra.

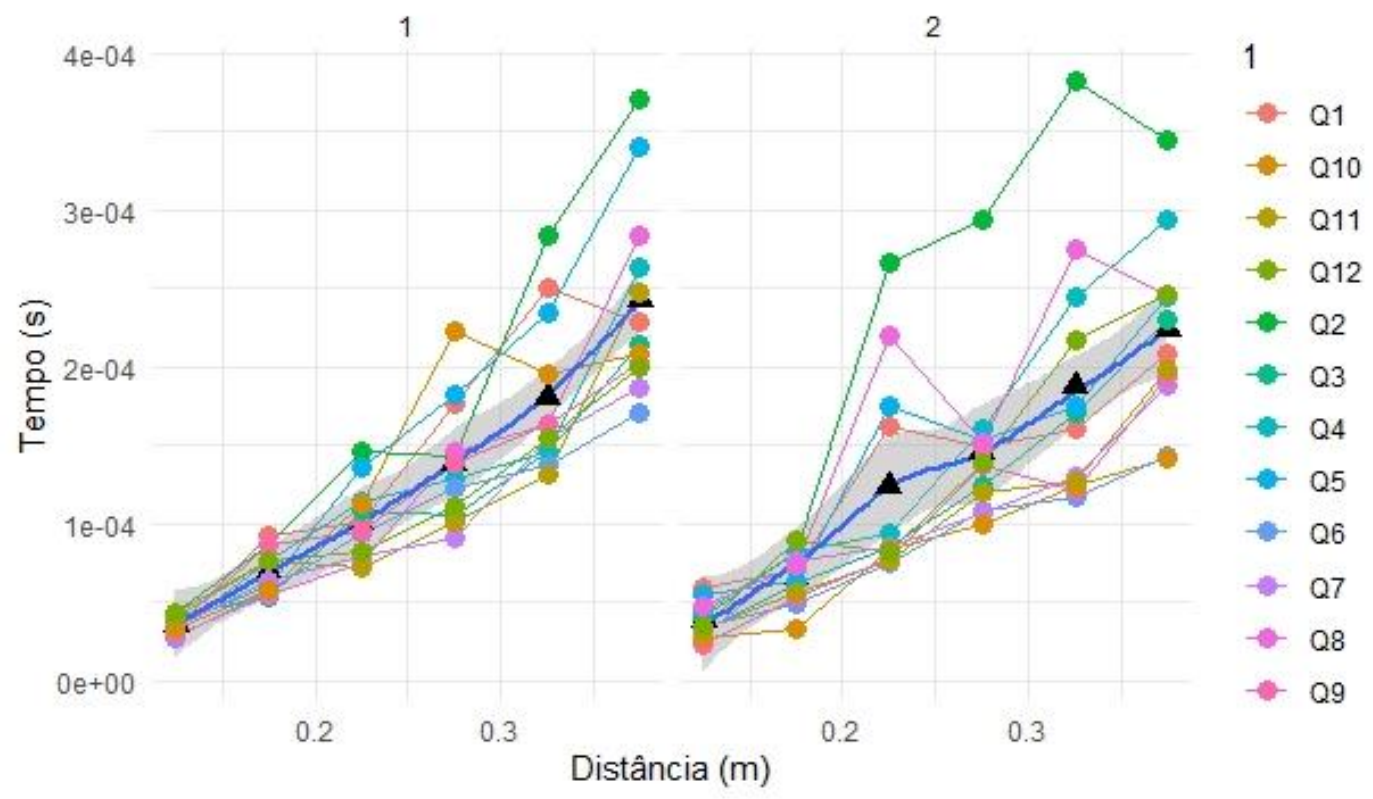

Figura 8: Perfis dos quadrantes com curva suavizada. 
Para tentar identificar, o comportamento das curvas e os quadrantes que perturbaram a curva suavizada, foi plotado o gráfico de perfis somente com alguns quadrantes que supostamente causaram a perturbação. Estes perfis podem ser vistos na Figura 9, em que, visualmente, tem-se Q8, Q5 e Q1 com comportamento semelhantes e com potencial para influenciar a tendência global. Para evidenciar a existência da perturbação causada pelas anomalias destes quadrantes, a Figura 10 mostra três curvas suavizadas, uma para o painel sem vazios, outra para o painel com vazios procurando separar aqueles que apareceram no gráfico de perfis com um comportamento aparentemente diferente dos demais e outro considerando somente os quadrantes que foram identificados como "perturbadores" da tendência global, de forma visual. Nota-se a partir desta figura que ao se retirar os quadrantes julgados como perturbadores, a curva suavizada para o painel com anomalias volta a ter uma tendência linear crescente, como é esperado, enquanto a curva a partir dos quadrantes perturbadores tem a sua curva suavizada bem distante de um comportamento linear.

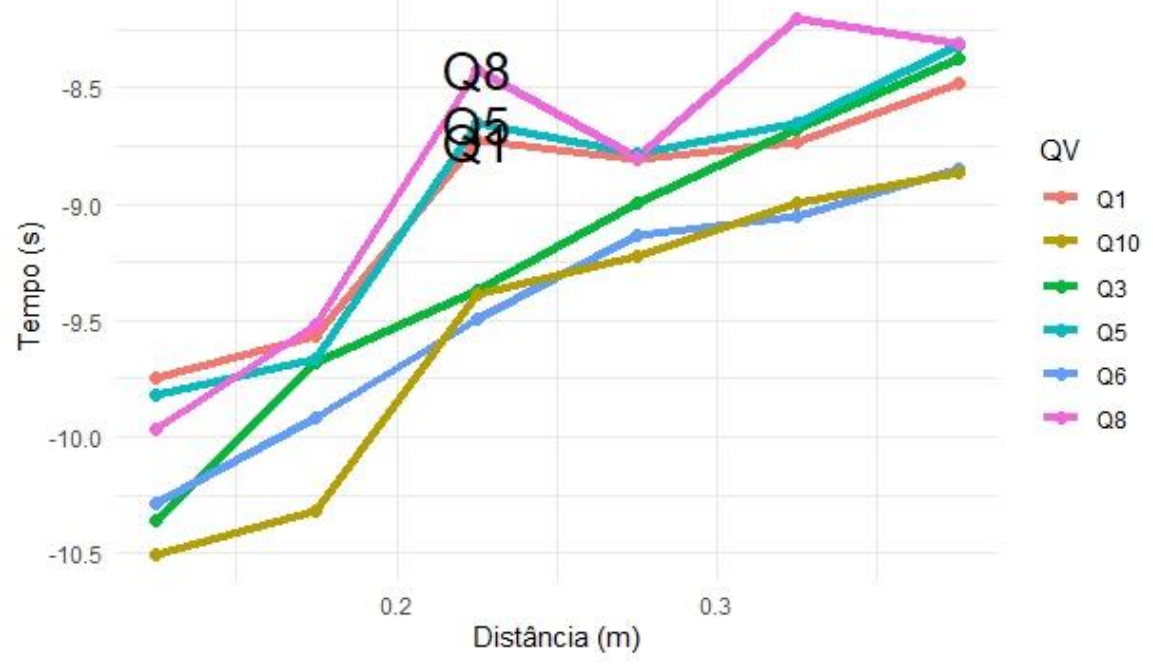

Figura 9: Perfis perturbadores, identificados visualmente.

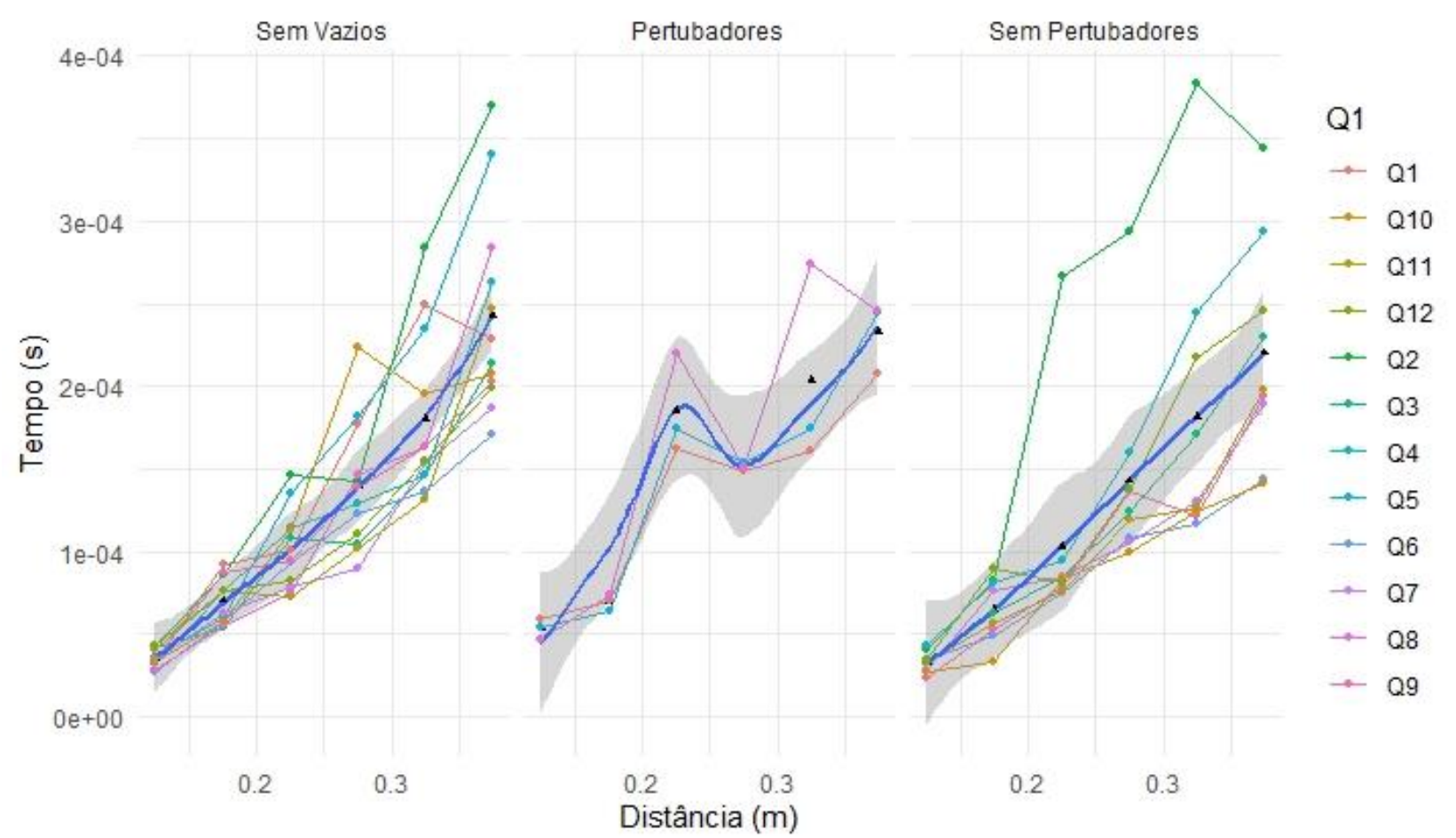

Figura 10: Curvas para os perfis classificados segundo painel e comportamento do perfil. 
As observações referentes a estas perturbações, em dados ultrassônicos de paredes com tijolos maciços, mostram indícios que as anomalias alteram o comportamento da onda nas regiões em que se encontram. Tal resultado preliminar pode nos dar possíveis pontos de fragilidade que podem conter vazios, de modo que a partir desta análise dos dados se tenha a identificação de potenciais locais de vulnerabilidade e heterogeneidade no interior das alvenarias. Em termos práticos espera-se que após processar os dados e analisar os gráficos seja possível ter evidências quanto as zonas da alvenaria que requerem maior atenção.

Outra vantagem da aplicação dessas ferramentas estatísticas é a possibilidade de visualização conjunta dos quadrantes, sendo possível a identificação de como as medições de um quadrante estão influenciando os dados globais do ensaio, sendo possível a distinção daqueles com o comportamento divergente, é importante destacar que as análises dos gráficos apenas nos dão indícios dos quadrantes com anomalias, não sendo necessariamente um resultado definitivo.

Vale ressaltar que muito ainda precisa ser estudado para entender e interpretar as diferentes situações que podem ser encontradas e como cada uma delas afeta o tempo de propagação da onda, como a presença de outros materiais, revestimentos mal aderidos à superfície e as interfaces de tijolo e argamassa. Os resultados obtidos aqui são preliminares, mas a partir desses resultados pode-se avançar com outras metodologias estatísticas, como o emprego de métodos de classificação ou agrupamentos de perfis para identificar pontos em alvenarias que podem estar com sua estrutura danificada. Para isso, é necessário que se tenha um conhecimento aprofundado do comportamento do tempo de propagação.

\section{CONCLUSÕES}

Diante do exposto neste estudo, constatou-se experimentalmente a usabilidade dos ensaios não destrutivos aplicados na análise da condição de alvenarias maciças através da execução do ensaio de pulso ultrassônico em dois painéis, onde um deles possuía anomalias estruturais. Por meio deste foi observado que os vazios geram perturbações na média dos dados de tempo da propagação das ondas, bem como tornou-se possível identificar indícios do posicionamento das anomalias, no qual tais resultados experimentais podem vir a contribuir para melhor analisar ensaios em campo.

Esses resultados preliminares foram possíveis através de uma nova abordagem de estudo por meio do emprego de ferramentas estatísticas que tornaram possíveis a visualização do comportamento da onda nos quadrantes, além de verificar a influência de cada quadrante nas medições, de modo a separar os que possuíam comportamento perturbador. Essa aplicação vem agregar uma contribuição para uma nova perspectiva da interpretação de dados ultrassônicos para o meio acadêmico da construção civil, e espera-se que as observações deste trabalho possam auxiliar como ferramenta para o estudo e preservação das construções históricas.

Com base nesses resultados nota-se novos ramos de pesquisa para trabalhos futuros, como a aplicação desse modelo em dados de campo para determinação de vazios presentes na estrutura. A análise e separação de diferentes materiais empregados, onde partir dos perfis das curvas ultrassônicas pode ser possível determinar os locais de mudança de comportamento, que pode ser uma ferramenta útil para determinação dos diferentes períodos construtivos existentes na estrutura. Outro exemplo é o aprofundamento do estudo com a realização de mais amostras para constatar a maior fiabilidade dos dados obtidos.

\section{AGRADECIMENTOS}

Os autores deste trabalho agradecem ao Laboratório de Reabilitação e Durabilidade das Construções (LAREB) pelos recursos mobilizados e pelo apoio durante a pesquisa, bem como a Universidade Federal do Ceará (UFC) por disponibilizar o equipamento e a estrutura técnica e laboratorial.

\section{REFERÊNCIAS}

ASSOCIAÇÃO BRASILEIRA DE NORMAS TÉCNICAS. NBR 8802: Concreto endurecido - Determinação da velocidade de propagação de onda ultrassônica. Rio de Janeiro: ABNT, 2019.

ALVES, A. L. S. Proposição de um método de caracterização de alvenarias de edificações históricas por meio de avaliação ultrassônica. monografia, 2017. 
ARÊDE, A.; COSTA, A. Inspecção e diagnóstico estrutural de construções históricas algumas contribuições da FEUP. p. 55-88, 2002.

BINDA, L.; SAISI, A.; TIRABOSCHI, C. Investigation procedures for the diagnosis of historic masonries. Construction and Building Materials, v. 14, n. 4, p. 199-233, 2000.

BROEMELING, Lyle D. Bayesian methods for repeated measures. Chapman and Hall/CRC, 2015.

CLEVELAND, William S.; GROSSE, Eric; SHYU, William M. Local regression models. In: Statistical models in S. Routledge, 2017. p. 309-376.

DE SOUZA, Rui Barbosa et al. Influência das variáveis atmosféricas na degradação dos materiais da construção civil. REEC-Revista Eletrônica de Engenharia Civil, v. 13, n. 1, 2017.

FERREIRA, Ana Sofia; BRITO, J. A importância da reabilitação em Portugal e na UE. In: Congresso Construção. 2007.

LERSCH, Inês Martina. Contribuição para a identificação dos principais fatores e mecanismos de degradação em edificações do patrimônio cultural de Porto Alegre. 2003.

MESQUITA, E. et al. Non-destructive characterization of ancient clay brick walls by indirect ultrasonic measurements. Journal of Building Engineering, v. 19, n. January, p. 172-180, 2018.

MESQUITA et al. Caracterización, evaluación y recuperación estructural de edificios

históricos. Boletim técnico. 2017. Disponível em: < http://alconpat.org.br/wp-content/uploads/2012/09/B11Caracteriza\%C3\%A7\%C3\%A3o-avalia\%C3\%A7\%C3\%A3o-e-recupera\%C3\%A7\%C3\%A3o-estrutural-de-

constru\%C3\%A7\%C3\%B5es-hist\%C3\%B3ricas.pdf>. Acesso em: 1 dez. 2019.

R CORE TEAM. R: A Language and Environment for Statistical Computing. R Foundation for Statistical Computing, Vienna, Austria, 2019.

ROQUE, João C. Almendra; LOURENÇO, Paulo B. Caracterização mecânica de paredes antigas de alvenaria: um caso de estudo no centro histórico de Bragança. Engenharia Civil, 2003.

TAVARES, Sinthya Goncalves. Desenvolvimento de uma metodologia para aplicação de ensaios térmicos não destrutivos na avaliação da integridade de obras de arte. 2006.

WENDRICH, A. et al. Location of voids in masonry structures by using radar and ultrasonic traveltime tomography. Proc. 9th European Conference on NDT, 25-29 Sept. 2006, Berlin, n. January, p. 1-11, 2006.

WICKHAM, Hadley. ggplot2: elegant graphics for data analysis. Springer, 2016. 